\title{
Hohenberg-Kohn Theory Including Spin Magnetism and Magnetic Fields
}

\author{
WALTER KOHN, ${ }^{1}$ ANDREAS SAVIN, ${ }^{2}$ CARSTEN A. ULLRICH ${ }^{3}$ \\ ${ }^{1}$ Departments of Physics and Chemistry, University of California, Santa Barbara, California 93106, USA \\ ${ }^{2}$ Laboratoire de Chimie Théorique CNRS et Université Pierre et Marie Curie, F-75252 Paris, France \\ ${ }^{3}$ Department of Physics, University of Missouri, Rolla, Missouri 65409, USA
}

Received 13 February 2004; accepted 19 March 2004

Published online 15 July 2004 in Wiley InterScience (www.interscience.wiley.com). DOI 10.1002/qua.20163

\begin{abstract}
Beginning with work by U. von Barth and L. Hedin in 1972 and continuing with recent papers in 2001 by K. Capelle and G. Vignale and by H. Eschrig and W. E. Pickett, questions have been raised about the meaning and validity of Hohenberg-Kohn theory when spin magnetism and/or magnetic fields are present. This article offers clarifications of some of these questions. In particular, it also concludes that these questions do not affect spin-density functional theory for nondegenerate ground states as currently practiced. (c) 2004 Wiley Periodicals, Inc. Int J Quantum Chem 100: 20-21, 2004
\end{abstract}

$\mathbf{T}$ he original Hohenberg-Kohn (HK) theorem [1] dealt with nonmagnetic, nondegenerate ground states of electrons in the presence of an external scalar potential $v(r)$. Under the tacit assumption that two different potentials $v(r)$ and $v^{\prime}(r)$ lead to different ground states $\Psi$ and $\Psi^{\prime}$ (physically irrelevant phase factors, $e^{i \theta}$ and $e^{i \theta^{\prime}}$, are disregarded), unless $v^{\prime}=v+C$, it was concluded that the density $n(r)$ of a nonmagnetic, nondegenerate ground state uniquely determines the scalar potential $v(r)$, apart from an arbitrary additive constant $C$.

In the following decade, similar considerations were applied to electronic ground states, including spin magnetism, $\vec{m}(r)$, and/or external magnetic fields, $\vec{B}(r)$, interacting with $\vec{m}(r)$, by Kohn and Sham [2] and by von Barth and Hedin [3]. The latter

\footnotetext{
Correspondence to: W. Kohn; e-mail: kohn@physics.ucsb.edu
}

investigators showed, for the case of a single particle, that the "4-density"

$$
\nu(r) \equiv[n(r), \vec{m}(r)]
$$

does not uniquely determine the "4-potential"

$$
w(r) \equiv[v(r), \vec{B}(r)]
$$

but only to within the following additive quantity:

$$
\Delta w(r)=\lambda u(r)\left[1, \vec{m}(r) /|\vec{m}(r)|^{2}\right]
$$

where $u(r)$ is an arbitrary function of $r$, provided that $\lambda$ is sufficiently small. Thus, in the presence of finite $\vec{m}(r)$ and / or $\vec{B}(r)$ the degree of nonuniqueness of $v(r)$ and $\vec{B}(r)$ is much "richer" than the "trivial" 
additive constant $C$ for $v(r)$ for the nonmagnetic systems considered by HK.

This theme was recently carried much further by K. Capelle and G. Vignale [4], as well as by H. Eschrig and W. E. Pickett [5]. These investigators considered many-electron systems with spin magnetism and magnetic fields and pointed out the existence of various rather simple ambiguities. In addition, Eschrig and Pickett displayed a very "nontrivial" type of ambiguity. These reports left many readers with the impression that "the HK theorem breaks down" when the coupling of a magnetic field $\vec{B}$ with spin magnetism is included.

In the present study, we aim to clarify the situation by a "deconstruction," expressing the HK theorem for nondegenerate ground states, in the presence or absence of $\vec{m}(r)$ and / or $\vec{B}(r)$, as consisting of two parts:

HK Theorem, Part I: A given physical 4-density $\nu(r)$ uniquely determines the ground state $\Psi$, apart from a trivial, unphysical phase-factor $e^{i \theta}$. This theorem, not subject to any other conditions, guarantees that any property of the system expressible in terms of the ground state only, without explicit recourse to the system's external 4-potential, $w(r)$, is a functional of the 4-density $\nu(r)$. For example, the two-particle correlation function $g\left(r_{1}, r_{2}\right)$, is implicit in the 4-density $\nu(r)$.

The proof of HK I uses the Rayleigh-Ritz variational principle for ground states (see ref. [1]).

HK Theorem, Part II: A 4-potential $w^{\prime}(r)$ leads to the same 4-density $\nu(r)$ and ground state $\Psi$ as a given 4-potential $w(r)$, if and only if their difference satisfies the condition

$$
\Delta W \Psi=\tilde{C} \Psi,
$$

where $\Delta W$ is the one-particle operator corresponding to $\Delta w(r)$ and $\tilde{C}$ is a sufficiently small constant.
Note that the addition of $\Delta W$ to the Hamiltonian $H$ does not perturb the ground-state $\Psi$, just as the addition of a constant $C$ to the scalar potential $v(r)$ does not lead to a perturbed ground state in the nonmagnetic case of HK. Thus, for the ground-state $\Psi, \Delta W$ is a "phantom perturbation." (However, except for the case $\Delta W=C, \Delta W$ clearly does perturb at least some excited states.) All the ambiguities in $w(r)$ of refs. [4] and [5] are easily seen to be phantom perturbations, with the exception of the nontrivial phantom perturbation in ref. [5].

Do these phantom perturbations cause problems to practical spin-density functional theory (DFT) for nondegenerate ground states? In our opinion, no. For, within that context, $v(r)$ and $\vec{B}(r)$ are given and not ambiguous; and the kinetic energy, classical Coulomb interaction, and exchange correlation energies, $T, U$, and $E_{x c}$ are defined in terms of $\Psi$ but independently of $v(r)$ and $\vec{B}(r)$. Hence, HK I confirms that $T, U$, and $E_{x c}$ are functionals of $n(r)$ and $\vec{m}(r)$ and HK II does not come into play.

A more complete manuscript, including proofs and a number of illustrations, is in preparation.

\section{ACKNOWLEDGMENT}

Support by NSF grants 0313980 and by 9976457 is gratefully acknowledged.

\section{References}

1. Hohenberg, P.; Kohn, W. Phys Rev 1964, 136, B864.

2. Kohn, W.; Sham, L. J. Phys Rev A 1965, 140, 1133.

3. von Barth, U.; Hedin, L. J Phys C 1972, 5, 1629.

4. Capelle, K.; Vignale, G. Phys Rev Lett 2001, 86, 5546.

5. Eschrig, H.; Pickett, W. E. Solid State Commun 2001, 118, 123. 